\title{
An Investigation of an Unbiased Correction for Heteroskedasticity and the Effects of Misspecifying the Skedastic Function
}

\author{
David A. Belsley \\ Boston College \\ February 23, 2000
}

\begin{abstract}
The traditional three-step procedure for correcting for heteroskedasticity uses a consistent but biased estimator for the variances $\boldsymbol{\sigma}_{t}^{2}$ in enacting the second step. An estimator is developed here that is umbiased in the presence of heteroskedasticity. Its behavior is examined along with the traditional estimator and another known to be unbiased in the absence of heteroskedasticity. The behavior of these corrective methods is also examined when the form and arguments of the skedastic function are misspecified. This is accomplished using Monte Carlo studies of several situations of interest.
\end{abstract}

\section{Introduction}

Heteroskedasticity has occupied econometricians for a long time, and with good reason, for, as we shall see, there are situations in which its presence, even in small samples, can render ordinary least squares (OLS) highly inefficient. We consider the regression context

$$
\mathbf{y}=\mathbf{X} \boldsymbol{\beta}+\varepsilon, \quad E \varepsilon \varepsilon^{T}=\boldsymbol{\Sigma}
$$

where $\mathbf{y}$ is a $T$-vector, $\mathbf{X}$ is a $T \times K$ data matrix, $\boldsymbol{\beta}$ is a $K$-vector of unknown parameters and $\boldsymbol{\Sigma} \equiv$ $\operatorname{diag}\left(\sigma_{1}^{2}, \cdots, \sigma_{T}^{2}\right)$. A function $g_{t}(\mathbf{z}, \boldsymbol{\alpha})$ specifying the $\sigma_{t}^{2}$ as functions of variates $\mathbf{z}$ and parameters $\boldsymbol{\alpha}$ is called the skedastic function, and we shall assume it takes the form

$$
E \varepsilon_{t}^{2} \equiv \sigma_{t}^{2}=g\left(\mathbf{z}_{t}^{T} \boldsymbol{\alpha}\right)
$$

where $\mathbf{z}_{t}^{T}$ is the $t^{\text {th }}$ row of $\mathbf{Z}$, a $T \times p$ matrix of data that could include columns of $\mathbf{X}, \boldsymbol{\alpha}$ is a $p$-vector of unknown parameters, and, while the argument of $g$ is linear in $\alpha, g$ itself can be any twice continuously differentiable function $\left(g \in C^{2}\right)$.

Of the two practical issues associated with heteroskedasticity, testing and correction, the former has of late been put on quite solid grounds with Davidson and MacKinnon's (1993) test based on artificial regressions (see also MacKinnon (1992)). Earlier tests were either inefficient (Goldfeld-Quandt, (1965)) or required an exact specification of the skedastic function $g$ (Breusch and Pagan (1979), Glejser (1969)) 
and possibly an assumption of Normality. By contrast, the test based on artificial regressions requires no assumtion of Normality, is the same regardless of the form of $g$ in (2) as long as it is $C^{2}$, and, as such, has admirable generality. However, if one rejects the null of homoskedasticity using this test, then any corrective action will require a full specification of the skedastic function $g$.

In usual practice, corrective action is taken using a three-step procedure. In the first, OLS of $\mathbf{y}$ on $\mathbf{X}$ is run to obtain residuals $\mathbf{e}=\left(e_{1}, \ldots, e_{T}\right)^{T}$, which are recognized to be consistent estimators of the $\boldsymbol{\varepsilon}_{t}$. In step two, a regression based on a relation between $e_{t}^{2}$ and $g\left(\mathbf{z}_{t}^{T} \boldsymbol{\alpha}\right)$ is used to obtain an estimate $\hat{\boldsymbol{\alpha}}$ of $\boldsymbol{\alpha}$, from which, in step three, correction takes place using feasible generalized least squares (FGLS) in the form of

$$
\mathbf{b}_{F G L S}=\left(\mathbf{X}^{T} \hat{\boldsymbol{\Sigma}}^{-1} \mathbf{X}\right)^{-1} \mathbf{X}^{T} \hat{\boldsymbol{\Sigma}}^{-1} \mathbf{y}
$$

where $\hat{\boldsymbol{\Sigma}} \equiv \operatorname{diag}\left(g\left(\mathbf{z}_{1}^{T} \hat{\boldsymbol{\alpha}}\right), \ldots, g\left(\mathbf{z}_{T}^{T} \hat{\boldsymbol{\alpha}}\right)\right)$. Three convenient forms for $g$, assumed in Breusch and Pagan (1979), are linear, square, and exponential, i.e., $\left(\mathbf{z}_{t}^{T} \boldsymbol{\alpha}\right),\left(\mathbf{z}_{t}^{T} \boldsymbol{\alpha}\right)^{2}, E^{\mathbf{z}_{t}^{T} \boldsymbol{\alpha}}$. In these cases, the second step, estimating $\alpha$, is carried out by the following regressions, respectively:

$$
\begin{aligned}
\text { linear }\left(\mathbf{z}_{\mathrm{t}}^{\mathrm{T}} \boldsymbol{\alpha}\right): & e_{t}^{2}=\mathbf{z}_{t}^{T} \boldsymbol{\alpha}+v_{t} \\
\text { square }\left(\mathbf{z}_{\mathrm{t}}^{\mathrm{T}} \boldsymbol{\alpha}\right)^{2}: & \left|e_{t}\right|=\mathbf{z}_{t}^{T} \boldsymbol{\alpha}+v_{t} \\
\text { exponential } \mathrm{E}^{\mathbf{z}_{\mathrm{t}}^{\mathrm{T}} \boldsymbol{\alpha}:} & \ln e_{t}^{2}=\mathbf{z}_{t}^{T} \boldsymbol{\alpha}+v_{t}
\end{aligned}
$$

In this paper, I exercise these corrective procedures by Monte Carlo studies in various situations of interest. I also add to the literature a form for the regressors in step two that is potentially better suited to small-samples. Whereas the $e_{t}^{2}$ are consistent estimates of the $\boldsymbol{\sigma}_{t}^{2}=g\left(\mathbf{z}_{t}^{T} \boldsymbol{\alpha}\right)$, they are not unbiased. Under the null of homoskedasticity, it is well known that $\tilde{e}_{t}^{2} \equiv e_{t}^{2} /\left(1-h_{t}\right)$ is an unbiased estimator of $E \varepsilon_{t}^{2}=\boldsymbol{\sigma}_{t}^{2} \equiv \boldsymbol{\sigma}^{2}$ for all $t$, where $h_{t}$ is the $t^{\text {th }}$ diagonal of the projection matrix $\mathbf{H} \equiv \mathbf{X}\left(\mathbf{X}^{T} \mathbf{X}\right)^{-1} \mathbf{X}^{T}$. Of course, when correcting for heteroskedasticity, this null has been rejected. So, in the next section, I derive an unbiased estimator $\hat{e}_{t}^{2}$ of $\boldsymbol{\sigma}_{t}^{2}$ when there is heteroskedasticity. In the Monte Carlo studies of subsequent sections, the behaviors of three regressors for step-two, $e_{t}^{2}, \tilde{e}_{t}^{2}$, and $\hat{e}_{t}^{2}$, are compared. Unfortunately, we shall see that the new estimator $\hat{e}_{t}^{2}$ does not fare as well as its author would have hoped.

In the next section, the unbiased estimator $\hat{e}_{t}^{2}$ of $\sigma_{t}^{2}$ is derived. In Section 3 , the three test data sets are described along with an overview of the Monte Carlo procedure employed. Section 4 reports the basic results. Section 5 examines an important special case where the heteroskedasticity is proportionate to a single variate. Section 6 examines the behavior of the corrective methods when the skedastic function is misspecified. Conclusions are offered in Section 7 . 


\section{An Unbiased Estimator of $\sigma_{t}^{2}$.}

It is well known that, if one estimates (1) by OLS, the regression residuals e are related to the disturbance term $\varepsilon$ by

$$
\mathbf{e}=\left[\mathbf{I}-\mathbf{X}\left(\mathbf{X}^{T} \mathbf{X}\right)^{-1} \mathbf{X}^{T}\right] \varepsilon \equiv[\mathbf{I}-\mathbf{H}] \varepsilon \equiv \mathbf{M} \varepsilon
$$

where the projection matrices $\mathbf{H}$ and $\mathbf{M}$ are defined by context. And when $\varepsilon$ is spherically distributed, i.e., $\boldsymbol{\Sigma}=\sigma^{2} \mathbf{I}$, we have

$$
V(\mathbf{e}) \equiv E \mathbf{e e}^{T}=E \mathbf{M} \varepsilon \varepsilon^{T} \mathbf{M}=\sigma^{2} \mathbf{M}
$$

the last equality holding due to the idempotency of $\mathbf{M}$. Thus, for the $t^{\text {th }}$ element of $\mathbf{e}$, we have

$$
E e_{t}^{2}=\left(1-h_{t}\right) \sigma^{2}
$$

where $h_{t}$ is the $t^{\text {th }}$ diagonal element of $\mathbf{H}=\mathbf{X}\left(\mathbf{X}^{T} \mathbf{X}\right)^{-1} \mathbf{X}^{T}$. Thus

$$
\tilde{e}_{t}^{2} \equiv \frac{e_{t}^{2}}{\left(1-h_{t}\right)}
$$

provides an unbiased estimator of $\sigma^{2}$ for all $t$ in the traditional OLS context.

In the event, however, that there is heteroskedasticity and $\boldsymbol{\Sigma}=\operatorname{diag}\left(\boldsymbol{\sigma}_{1}^{2}, \cdots, \boldsymbol{\sigma}_{T}^{2}\right)$, we have

$$
\begin{aligned}
V(\mathbf{e}) & \equiv E \mathbf{e e}^{T}=E \mathbf{M} \varepsilon \varepsilon^{T} \mathbf{M}=\mathbf{M} \boldsymbol{\Sigma} \mathbf{M} \\
& =\boldsymbol{\Sigma}-\mathbf{\Sigma} \mathbf{H}-\mathbf{H} \boldsymbol{\Sigma}+\mathbf{H} \boldsymbol{\Sigma} \mathbf{H},
\end{aligned}
$$

and bit of algebraic manipulation verifies that

$$
\begin{aligned}
E e_{t}^{2} & =\sigma_{t}^{2}-2 h_{t t} \sigma_{t}^{2}+\left(h_{t 1}^{2} \sigma_{1}^{2}+\cdots+h_{t t}^{2} \sigma_{t}^{2}\right) \\
& =\sigma_{t}^{2}-2 h_{t t} \sigma_{t}^{2}+h_{t t} \sigma_{t}^{2} \frac{\left(h_{t 1}^{2} \sigma_{1}^{2}+\cdots+h_{t t}^{2} \sigma_{t}^{2}\right)}{h_{t t}^{2} \sigma_{t}^{2}} \\
& =\sigma_{t}^{2}\left(1+h_{t}\left(\psi_{t}-2\right)\right)
\end{aligned}
$$

where, in line with convention, we rename $h_{t t}$ to be $h_{t}$, and define

$$
\psi_{t} \equiv \frac{\sum h_{t j}^{2} \sigma_{j}^{2}}{h_{t} \sigma_{t}^{2}} .
$$

This last term will be recognized for each $t$ to be a weighted average of the $\left(\sigma_{j}^{2} / \sigma_{t}^{2}\right)$ since $\sum_{j=1}^{T} h_{t j}^{2}=h_{t}$ due to the idempotency of $\mathbf{H}$.

Thus, in the case of heteroskedasticity,

$$
\frac{e_{t}^{2}}{\left(1+h_{t}\left(\psi_{t}-2\right)\right)}
$$


is an unbiased estimator of $\sigma_{t}^{2}$. Unfortunately, $\psi_{t}$ contains the unknown $\sigma_{t}^{2}$ and so cannot be used as stands, but it can be estimated in as part of a four-step procedure. Thus, in step 1, regress $\mathbf{y}$ on $\mathbf{X}$ to obtain the OLS residuals e. In step 2, first use the $\mathbf{e}^{2}$ in the normal fashion to obtain an estimate $\hat{\alpha}$ for the argument of the particular skedastic function $g$ being assumed. Then use the resulting $g\left(\mathbf{z}_{t}^{T} \hat{\alpha}\right)$ to provide estimates $\hat{\sigma}_{t}^{2}$ of the $\sigma_{t}^{2}$, which in turn can be used in (11) to provide estimates of the $\psi_{t}$ as

$$
\hat{\psi}_{t} \equiv \frac{\sum h_{t j}^{2} \hat{\sigma}_{j}^{2}}{h_{t} \hat{\sigma}_{t}^{2}}
$$

From these we derive the transformed OLS residual squares

$$
\hat{e}_{t}^{2} \equiv \frac{e_{t}^{2}}{\left(1+h_{t}\left(\hat{\psi}_{t}-2\right)\right)}
$$

to be used as estimates of (12). In step 3, the $\hat{e}_{t}^{2}$ are used, again in the normal fashion, to obtain an estimate $\hat{\hat{\alpha}}$ for the argument of the particular skedastic function $g$ being assumed. Then, in step 4, $\hat{\boldsymbol{\Sigma}}$ is estimated as the diagonal matrix of the $g\left(\mathbf{z}_{t}^{T} \hat{\hat{\alpha}}\right)$ and used in (3) to obtain the final FGLS estimator of $\boldsymbol{\beta}$.

We turn now to Monte Carlo studies to see just how well this rather busy procedure performs as well as to learn more in general about the process of correcting for heteroskedasticity.

\section{The Data and Monte Carlo Procedure}

Three basic data sets are used for the Monte Carlo experiments that follow.

3.1 The Data Sets. The first is a real economic data set taken from pages 365-66 of Pindyck and Rubinfeld (1991) and comprises 114 observations on three series giving monthly prices, production, and stocks of heating oil over the period 1979-01 - 1988-06. These series, along with an intercept term, become the $\mathbf{X}$ data for a set of experiments that will be denoted "Rubin." These data are relatively smooth, showing some mild cyclical activity but no major trends or discrepancies of level.

By contrast, the second data set, which will be referred to as "Mix," comprises three series generated to be a mixture of large and small values. This is seen from the following Mathematica code used to generate a data set of size $n$.

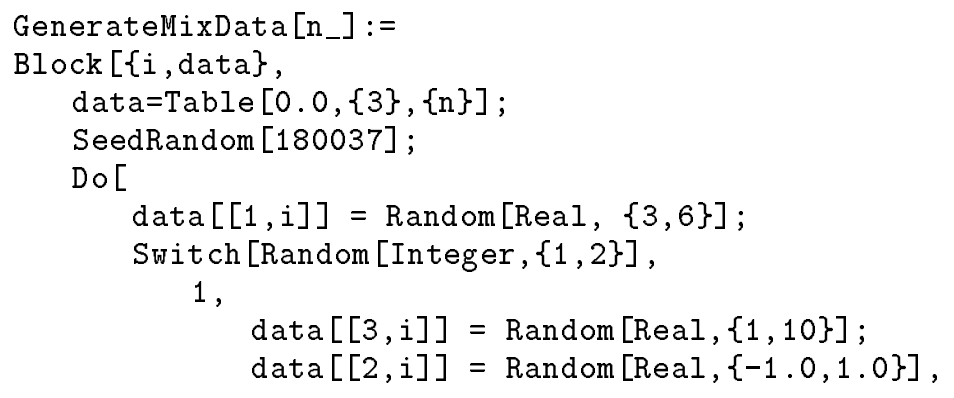




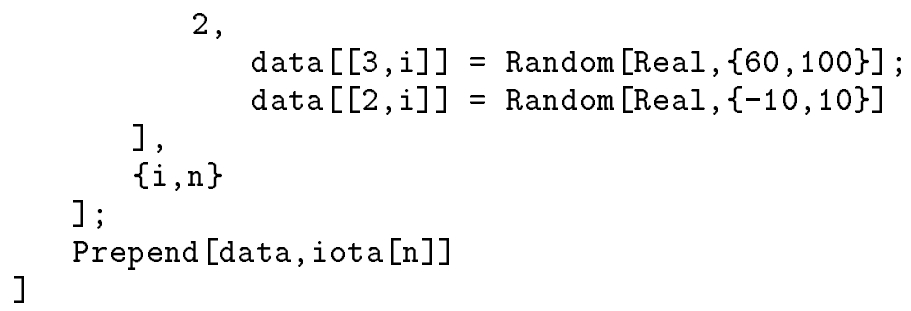

The $i^{\text {th }}$ observation for the three variates is drawn randomly. However, while variate one comes from the same population across all observations, variates two and three are chosen randomly to be from either a "large-" or a "small-" magnitude population. A constant term is prepended in the last line, iota[n] being a vector of $n$ ones. The seed is fixed at the outset so that the data set is repeatedly reproducable. This population allows one to explore the behavior of heteroskedasticity that is related to the magnitude of the entities involved, as might be the case, for example, if one specifies the variance of investment behavior to vary with the capital value of the firm.

The third data set is generated to have a trended element, and is referred to, not surprisingly, as "Trend." Its construction can be seen from the following Mathematica code to generate a set of Trend data of size $n$.

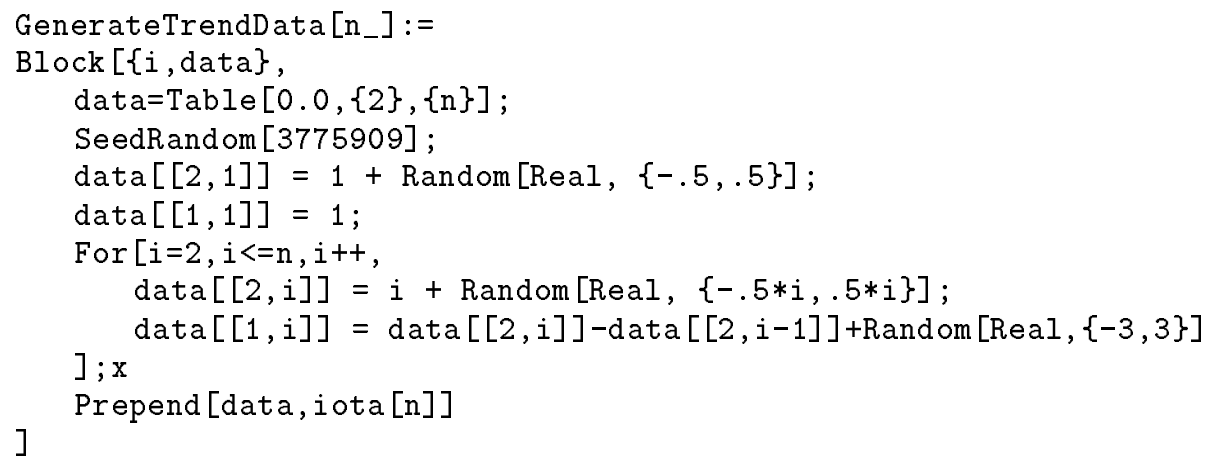

Starting with initial conditions for observation 1 , the second variate in subsequent observations increases with a strong trend component, while the first variate moves roughly as a first-difference. Again, a constant variate of ones is prepended and an initial seed guarantees reproducibility of the data set for fixed $n$. This pattern is typical of many economic time series, and, unlike the Rubin and Mix data, is nonstationary.

3.2 The Monte Carlo Procedure. For each of the Monte Carlo experiments, the $\mathbf{z}_{t}$ and $\alpha$ are first defined for use in the three skedastic functions (4). For each skedastic function, 5000 replications are made for each of three sample sizes, 20, 114, and 456, representing small, medium, and moderately large data sets. In each case, the $\mathbf{y}$ data are generated from (1) with $\varepsilon$ being generated as independent Normals with mean zero and variances determined by the appropriate skedastic function. For each replication, estimates of $\boldsymbol{\beta}$ are obtained for each of four correction methods: 
(1) no correction for heteroskedasticity, $\hat{\boldsymbol{\Sigma}}$ in (3) is $\sigma^{2} \mathbf{I}$ : OLS of $\mathbf{y}$ on $\mathbf{X}$,

(2) FGLS, where $\hat{\boldsymbol{\Sigma}}$ is estimated in the second step using $e_{t}^{2}$ from (5),

(3) FGLS, where $\hat{\boldsymbol{\Sigma}}$ is estimated in the second step using $\tilde{e}_{t}^{2}$ from (8), and

(4) FGLS, where $\hat{\boldsymbol{\Sigma}}$ is estimated in the third step using $\hat{\boldsymbol{e}}_{t}^{2}$ from (14).

In the basic experiments, the corrective model employed is the one appropriate to the way the data were generated; that is, if the data were generated using a square skedastic model (4b), then the square model is employed in the estimation of $\boldsymbol{\alpha}$, etc. Later experiments explore what happens if the form of the corrective model is misspecified. However, the basic experiments do examine what happens if the variates involved in the generation of the heteroskedasticity (the arguments $\mathbf{z}_{t}$ of the skedastic function) are not known exactly. In the Rubin model, the $\mathbf{z}_{t}$ and $\mathbf{x}_{t}$ data are the same; that is, all the variates in $\mathbf{X}$ are used in generating the heteroskedasticity. In the Mix and the Trend models, however, only a subset of the $\mathbf{X}$ data is used for the $\mathbf{z}_{t}$ data. For these models, separate Monte Carlo experiments are conducted under the 'ignorance assumption,' that all the $\mathbf{X}$ data could be involved in $\mathbf{z}_{t}$ when correcting for the heteroskedasticity, and under the 'knowledgable assumption,' that the exact specification of $\mathbf{z}_{t}$ is known.

There are Monte Carlo runs, then, for each of three different skedastic functions (4), three different sample sizes $(20,114,456)$, and four different corrective methods (uncorrected, $e_{t}^{2}, \tilde{e}_{t}^{2}$, and $\hat{e}_{t}^{2}$ ). Each run results in 5000 estimates $\mathbf{b}_{i}$ of $\boldsymbol{\beta}$, which can be used to calculate a sample mean-square-error matrix

$$
\mathbf{S} \equiv \frac{1}{5000} \sum_{i=1}^{5000}\left[\mathbf{b}_{i}-\boldsymbol{\beta}\right]\left[\mathbf{b}_{i}-\boldsymbol{\beta}\right]^{T} .
$$

A sample covariance matrix, using $\overline{\mathbf{b}}$ instead of $\boldsymbol{\beta}$ could have been used instead, but would have produced no meaningful differences in result. In each and every case, the sample mean $\overline{\mathbf{b}}$ was an excellent estimate of $\boldsymbol{\beta}$ to within three or four significant digits - consonant with theory, which notes these estimators all to be unbiased. For each situation, we can also calculate the exact variance-covariance matrix of the estimator in question, i.e.,

$$
\mathbf{V} \equiv\left(\sum_{t=1}^{T} \sigma_{t}^{-2} \mathbf{x}_{t} \mathbf{x}_{t}^{T}\right)^{-1}
$$

where $\sigma_{t}^{2}$ is defined for the appropriate skedastic function by (2), $\mathbf{x}_{t}^{T}$ is the $t^{\text {th }}$ row of $\mathbf{X}$, and $T$ is 20,114 , or 456, depending on sample size. Evaluation of the different corrective methods is then based on comparing the Relative Generalized Mean Square Error (RGMSE), that is, comparing the ratio of determinants 
As is well known, special care is required in correcting for heteroskedasticity in the linear model. It is quite possible for particular instances to produce very small or even negative estimates of the variance. Some effort is made to pick $\boldsymbol{\alpha}$ s to minimize this effect. However, the skedastic function is defined for this model to set $\hat{\sigma}_{t}^{2}=.03$ for all such occurances less than .03 .

In the tables that follow, the RGMSEs for the four different correction methods (no correction, $e_{t}^{2}, \tilde{e}_{t}^{2}$, and $\hat{e}_{t}^{2}$ ) are listed in each case in order from best (least) to worst (largest). Since the corrective methods can be ordered differently from situation to situation, it proves necessary to have a means for identifying a RGMSE value with a particular corrective method. To this end, the following key is used: the RGMSE values for the uncorrected OLS method are printed using the regular roman type face, the method using $e_{t}^{2}$ has values printed in the italic face, the method using $\tilde{e}_{t}^{2}$ has values in bold face, and the method using $\hat{e}_{t}^{2}$ has values in a different font altogether looking like this. A key (uncorrected, $e_{t}^{2} \cdot \tilde{\mathbf{e}}_{t}^{2}, \hat{\mathrm{e}}^{2}$ ) is provided at the bottom of each table to facilitate this identification.

\section{The Basic Results}

In this section we exmine the basic results for the three data sets, Rubin, Mix, and Trend.

4.1 Rubin Data. We begin with the basic Rubin results shown in Table 1. In this case, the $\mathbf{z}_{t}$ in (4) are the $\mathbf{x}_{t}$ (the rows of the Rubin $\mathbf{X}$ data), and $\boldsymbol{\alpha}=(1,-.01, .005, .02)$. Thus, all of the variates in $\mathbf{X}$ are involved in the determination of the skedasticity. Examine first the results for the square skedastic function. For the smallest sample size, 20, the uncorrected (simple OLS) method has the smallest RGMSE of 1.32, the method using $e_{t}^{2}$ is next best with a RGMSE of 1.84, the method using $\tilde{e}_{t}^{2}$ (a simple $h_{t}$ adjustment) follows with a RGMSE of 2.04, and the newly proposed method using $\hat{e}_{t}^{2}$ (additional $\psi_{t}$ adjustment) comes last with a RGMSE of 3.33. For a moderate sample size of 114, this order changes, and the $e_{t}^{2}$ and $\tilde{e}_{t}^{2}$ methods do best, followed closely by $\hat{e}_{t}^{2}$, and uncorrected OLS comes last. This order is preserved at the largest sample size of 456, but there is no practical difference among the three corrections, all of which outstrip uncorrected OLS. We can conclude in this instance that some correction is helpful for moderate to large sample sizes, but for small sample sizes either no correction or the simplest $e_{t}^{2}$ correction is best.

In the case of a linear skedastic function, a somewhat similar story arises, except that corrective efforts beyond uncorrected OLS do not help much until the largest sample size, and even here uncorrected OLS does realtively well. I would judge that correction for heteroskedasticity in this linear case is basically unnecessary. In any event, the simplest correction $e_{t}^{2}$ is better than the other two.

For the exponential case, uncorrected OLS still reigns for small sample sizes, where we note that the $\hat{e}_{t}^{2}$ correction is very poor indeed. For moderate to large sample sizes, however, correction helps considerably 
over OLS. While $\tilde{e}_{t}^{2}$ is best, $e_{t}^{2}$ is very close. Again, the simplest correction is adequate.

4.2 Mix Data. The basic Mix data results are reported in Table 2. In this case, the $\mathbf{z}_{t}$ that generate the heteroskeasticity in (4) include only the intercept and fourth variate of the $\mathbf{X}$ data, and $\boldsymbol{\alpha}=(1.0, .02)$. We recall that for these data, the fourth $\mathbf{X}$ variate comes from two separate populations, large and small, so that there will be 'high'- and 'low'-variance observations. Two sets of results are reported in Table 2. In the first set, labelled "All Variates," it is assumed that, in effecting the correction, the exact $\mathbf{X}$ variates used in generating the heteroskedasticity are not known, so the step estimating $\boldsymbol{\alpha}$ employs them all. This clearly should lead to a loss of power for the corrective procedure. In the second set, labelled "Exact Variates," it is assumed that the variates involved in generating the heteroskedasticity are known.

The All Variates results here for each skedastic function are very similar to those reported above for the basic Rubin data. For small samples, the uncorrected OLS method is best, although it is now somewhat more inefficient with a RGMSE of over two. This remains the case for the linear skedastic function in the moderate sample size of 114 , but, for all other cases, one or the other of the corrective methods proves best. Again, the $e_{t}^{2}$ method is either best or not far behind the $\tilde{e}_{t}^{2}$ method.

The Exact Variates results are a very different story. Here one or another of the corrective methods is best even with the smallest sample size of 20 . And, for the first time, the more sophisticated correction methods, $\tilde{e}_{t}^{2}$ and $\hat{e}_{t}^{2}$, become best for the larger samples sizes, but not by very much. Indeed, there is nothing in these results to indicate any real advantage to corrective methods beyond the simplest, $e_{t}^{2}$.

It would seem that, when one knows the exact nature of the skedastic function, i.e., its proper form and variate inclusion, that some correction is warranted over uncorrected OLS regardless of sample size. If the proper form for the skedastic function is known, but not the variate inclusion for $\mathbf{z}_{t}$, simple correction is warranted for moderate to large sample sizes, but uncorrected OLS is best for small samples.

4.3 Trend Data. The basic Trend data results are reported in Table 3. As for the Mix data, only a subset of the $\mathbf{X}$ data are used for the $\mathbf{z}_{t}$ in generating the heteroskedasticity: an intercept and the trended third variate; here $\boldsymbol{\alpha}=(1.0, .005)$. Again, results are reported for the inclusion of All Variates and for Exact Variates in effecting the step estimating $\boldsymbol{\alpha}$. The results for All Variates are very similar to those just reported for the Mix case above. Basically, uncorrected OLS is best for small samples (20), while some form of correction is beneficial for moderate to large samples. The correction that proves best varies a bit, but, for the most part, they all do pretty much equally well, so there is little reason to use anything beyond the simplest correction, $e_{t}^{2}$. A different story arises in the Exact Variates results. In the Mix case reported above, uncorrected OLS was dominated at all sample sizes. Here, however, we see that uncorrected OLS 
does very well for small and even moderate sample sizes. Only in the square model does correction help at the 114 sample size. By the time the sample size gets to 456 , however, correction of some sort is beneficial and notably superior to uncorrected OLS. The form of the correction again makes little difference.

\section{Proportionate Skedasticity Results}

In the preceding Monte Carlo studies, the heteroskedasticity is generated as a linear combination of two or more of the $\mathbf{X}$ variates, including an intercept term. In the studies reported here, the heteroskedasticity is generated (in the context of the Mix and Trend models) as a function of one of the $\mathbf{X}$ variates alone. This is a case often assumed in econometric analysis. For ease of reference, I shall refer to this situation as the proportionate case, but there would be a truly proportionate relation (for either variance or standard deviation) only for the square and linear models, not for the exponential model. In the Mix model, the heteroskedasticity is made to depend only on the fourth $\mathbf{X}$ variate, the one that aquires large and small values for different observations. In the Trend model, the heteroskedasticity depends only on the trended variate. Once again, in both these cases, the results are reported for using All Variates and Exact Variates in the step estimating $\boldsymbol{\alpha}$.

It should be noted that when the skedastic function is either square or linear in this proportionate case, the three corrective methods, $e_{t}^{2}, \hat{e}_{t}^{2}$, and $\hat{e}_{t}^{2}$, give exactly the same results. Thus only two RGMSE values are published for these situations, one for uncorrected OLS (in roman face) and one for the three corrections (in italic face).

5.1 Mix Data: Proportionate. The proportionate Mix data results are reported in Table 4. The results for All Variates in the square skedastic model are immediately remarkable. It is quite clear that correction for heteroskedasticity when the skedastic function is the popularly assumed square model can be highly problematic, even for large sample sizes, when there is ignorence of the exact variates involved in the generation of the heteroskedasticity. All three of the corrective methods as well as uncorrected OLS do very poorly, showing highly inflated GRMSEs. A glance at the results for Exact Variates shows that knowledge of the exact variate involved is very important in this case. With knowledge of the exact variate involvement, any of the three corrective methods is highly effective at any sample size. The uncorrected OLS results, of course, retain their same poor showing regardless of any such knowledge and are clearly to be avoided in the case of the proportionate square model. That better knowledge of variate involvement should produce lower-variance estimates for the corrective methods is to be expected, but I am surprised at the extent of the difference observed here and the fact that it appears to affect only the square model so.

No such problem plagues either the exponential or linear models. For the exponential model using All 
Variates, uncorrected OLS fares slightly better in the small sample, but fares worst for medium to large sample sizes, where $\tilde{e}_{t}^{2}$ does best — but only slightly so over the other corrective means. In the linear model, $\tilde{e}_{t}^{2}$ does best — but only marginally so as the sample size increases — and uncorrected OLS comes in last for all sample sizes.

When the exact variate is known, all the corrective methods prove superior to uncorrected OLS for all sample sizes, a result similar to what was found for the basic Mix data above. It is of interest to note that the most sophistocated correction, $\hat{e}_{t}^{2}$, does best for larger sample sizes, but only marginally so. For the small sample, both $\tilde{e}_{t}^{2}$ and $\hat{e}_{t}^{2}$ show some advantage. Again, however, the advantage is not so great as to warrant the added complication involved in their computation: the simple $e_{t}^{2}$ correction does well too.

It seems appropriate to conclude that, in the proportionate case, some correction is superior to uncorrected OLS, although in the square model, this is a superiority is that of bad against worse.

5.2 Trend Data: Proportionate. Table 5 presents the results for the Trend data with proportionate heteroskasticity. In this instance, there are three sets of results. The first is for All Variates (no restrictions on variate involvement in the step estimating $\boldsymbol{\alpha}$ ), the second set for Partial Variates (only the intercept and the trend variate are used in the step estimating $\boldsymbol{\alpha}$ ), and the third set for Exact Variates (only the trend variate is included in the $\boldsymbol{\alpha}$-estimating step). In addition, in the Exact Variate set, a second figure for the 456-observation sample size is included. This will be explained shortly.

We note again in the results for All Variates for the square model that the inefficiency of not knowing the proper variate involvement produces extreme results — and the variance increases with sample size. This is similar to the behavior for the square model for the Mix data. To examine this behavior, a Partial-Variate group is added, where the estimates of $\boldsymbol{\alpha}$ are made under the assumption that only the intercept and the trended variate are involved in the generation of the heteroskedasticity. This meaningful prior information helps considerably, and, for small to moderate samples the various corrective mechanisms are much better than uncorrected OLS. However, for large sample sizes, uncorrected OLS regains superiority, and, overall, the RGMSEs are very large. It is clear that the absence of exact knowledge of the variates involved in generating the heteroskedasticity hurts, particularly in the square model. Things settle down when the exact variate involvement is known, and all corrective methods prove superior to uncorrected OLS even at the smallest sample size. However, note that the RGMSE continues to grow with sample size. To examine whether this is the result of the nonstationary nature of the trend, the 456 sample results are re-examined by cycling the 114 data four times rather than by continuing the trend for all 456 observations. It can be seen here, and for the exponential and linear cases, that this does indeed check the growth of the RGMSE, but this statistic does not fall with sample size. 
The situation is much improved in both the exponential and linear models, where the results tend towards the patterns we have seen emerging in other situations: uncorrected OLS tends to be best for the small sample size, but correction seems warrented for medium and large samples sizes, and there is not much to suggest the superiority of any one corrective method over another.

\section{Misspecified Tests}

In the preceding runs, the corrective skedasticity functions are correctly specified to match the generating skedasticity function. That is, if the data are generated with, say, an exponential skedasticity function, the correction also employs an exponential function. The question naturally arises how the corrections fare if the form of the skedastic function is misspecified, and we examine this issue here for the three data sets. The data are generated in turn with one of the three skedasticity models while the corrections are effected with the other two.

6.1 Rubin Data: Misspecified Table 6 reports the results for misspecified skedasticity corrections in the context of the Rubin data. In the top tableu of the table are the results for the case where the data are generated with a square skedasticity model and corrected with exponential and linear skedasticity functions. The misspecification as exponential is seen to produce surprisingly good results for all sample sizes, but particularly for moderate and large sample sizes, where all three correction methods outperform uncorrected OLS. With the linear misspecification, uncorrected OLS surpasses all corrective methods until the largest sample size, at which point $e_{t}^{2}$ performs best, but not much better than uncorrected OLS.

When the data are generated with the exponential model, misspecification as square produces acceptable results. Again, uncorrected OLS seems best here until the largest sample size, where any of the corrective methods seem preferable. It is quite clear that no linear corrective method is desirable relative to uncorrected OLS.

When the data are generated with the linear model, uncorrected OLS again is preferable to either square or exponential misspecifications, but $e_{t}^{2}$ or $\tilde{e}_{t}^{2}$ are very close competitors in both cases. The general weight of the evidence is, not surprisingly, that one is better off using uncorrected OLS than in misspecifying the correction skedasticity function, although misspecifying the square with the exponential and vice versa is not too bad. Misspecifying either the square or exponential models with linear is clearly to be avoided, although misspecifying linear with either square or exponetial is not that bad.

6.2 Mix Data: Misspecified. Tables 7 and 8 examine the results of misspecifying the Mix data. In Table 7 , the skedasticity is generated using two variates, the intercept and the Mix variate, the variate that takes high and low values for different observations. These results are very similar to those reported above 
for the misspecifications with the Rubin data. Table 8 reports the results where the heteroskedasticity is generated only with the Mix variate, the so-called proportionate case. Here the results are quite different and warrant attention. The effects of misspecifying the square model as exponential are seen to be devastating. This is clearly a situation to be avoided. Misspecification as linear in this context is not good either, but not nearly as bad as exponential. Note that the three corrective methods produce the same results here.

The exponential model misspecified as square is also to be avoided. Here, however, uncorrected OLS does fairly well. The situation is not as bad with a linear misspecification, where uncorrected OLS seems to inflate RGMSE only by a factor of 2. The linear model misspecified as either square or exponential is again to be avoided, regardless of sample size.

6.3 Trend Data: Misspecified. Table 9 reports the results of the trend model with corrective misspecification. For these data, we examine only the proportionate case where the heteroskedasticity depends on the single trend variate. These results are seen to be very close to those for the proprotionate Mix data just reported in Table 8.

\section{Conclusions}

There are two areas of conclusions for this study: a comparison of the corrective methods $e_{t}^{2}, \tilde{e}_{t}^{2}$, and $\hat{e}_{t}^{2}$, and an examination of the effects of misspecifying the skedastic function. There is some interplay between the two.

7.1 Comparing the Corrective Methods. The following conclusions are indicated in comparing the behavior of the three corrective methods along with uncorrected OLS:

- For small samples (20 observations), uncorrected OLS is very acceptable and often dominates any form of correction. The only situation in which this may not the case is when the exact specification of the skedastic function, both its arguments and form, is known. By in large, correction is not indicated for small samples.

- For moderate and large sample sizes (114 and 456 observations) correction becomes worthwhile.

- When correction is worthwhile, however, there appears to be little value in using anything but the simplest correction $e_{t}^{2}$. When corrections based on either $\tilde{e}_{t}^{2}$ or $\hat{e}_{t}^{2}$ are better, they are typically marginally so. There is little reason to worry about having additional weapons in the arsenal.

- The previous conclusion is a rather discouraging result for the value of the new unbiased correction, $\hat{e}_{t}^{2}$. There are situatons in which this corrective method does best, but never better enough to warrant the extra effort in computing it. Basically, when uncorrected OLS does best, $\hat{e}_{t}^{2}$ tends to do worst, and when $\hat{e}_{t}^{2}$ does best, $e_{t}^{2}$ or $\tilde{e}_{t}^{2}$ are essentially just as good. 
7.2 Examining Misspecification of the Skedastic Function. The lessons here for practice, if any, are rather messy. There are two relevant types of misspecification: one can misspecify the variates that are involved in generating the heteroskedasticity, i.e., the arguments of the skedastic function, and one can misspecify the form of the skedastic function.

- The first form of misspecification has been examined in the context of the Mix and the Trend data, where results are given for the use of All Variates in the second/third-step estimates of $\alpha$ and for the use of Exact Variates. It is clear that the inclusion of irrelevant variates in this step can cause substantial inflation in the RGMSE for small and moderate sample sizes, and in some instances even in larger samples sizes. (This phenomenon seems, for some reason, to be particularly problematic for the square model with heteroskedasticity generated proportionately to a single variate — see Tables 4 and 5 - but estimated using All Variates.) It should be noted that this form of misspecification is examined only by including irrelevant variables, not by excluding relevant ones.

- The second type of misspecification, that of the form of the skedastic function, can be rather beneign in some situations and quite harmful in others. If the generation of the heteroskedasticity includes a constant component (an intercept term in $\boldsymbol{\alpha}$ ), the use of a misspecified skedastic function can be strangely forgiving. Only misspecifying exponentially generated heteroskedasticity as linear causes real problems. Indeed, functional misspecification seems to be most deleterious in the proprotionate case, when the actual heteroskedasticity generation involves but a single variable. Here the effects of the misspecification can be disasterous, even for large sample sizes. Further, it appears that there is no simple lesson, like "ignore correction unless you know for sure the proper form of the skedasticity function," for uncorrected OLS does not do well in the presence of a heteroskedasticity generated with a square skedastic function.

- Correction for heteroskedasticity clearly does best when both the proper arguments and form of the skedasticity function are known. But this is an empty conclusion since misspecification is probably the rule. Thus, further research is warranted in dealing with the issue. A specification test for the form of the skedasticity function is high on the list. The use of an encompassing model is an obvious thought, as would be a method that compares the estimated FGLS variances of $\mathbf{b}_{F G L S}$ in (3) across the different skedastic functions, picking the one with the best looking standard errors.

\section{Bibliography}

Belsley, D. A. (1995), "Doing Monte Carlo Studies with Mathematica: A Case Study Determining the Relative Power of Zero-Padding when Testing for Serial Correlation Using Artificial Regressions," forthcoming, Economic and Financial Modeling with Mathematica, Vol. II, Edited by Hal R. Varian, Telos, The Electronic Library of Science:Santa Clara, CA. 
Belsley, D. A. (1997), “A Small-Sample Correction for Testing for gth-Order Serial Correlation with Artificial Regressions," Computational Economics, 10, 197-229.

Breusch, T. S., and A. R. Pagan (1979), "A Simple Test for Heteroskedasticity and Random Coefficient Variation," Econometrica, 47, 1287-94.

Davidson, R, and J. G. MacKinnon (1993), Estimation and Inference in Econometrics, Oxford University Press: New York.

Glejser, H. (1969), “A New Test for Heteroskedasticity," Journal of the American Statistical Association, 64, 316-23.

Goldfeld, S., and R. E. Quandt (1965) "Some Tests for Homoskedasticity," Journal of the American Statistical Association, 60, 539-47.

MacKinnon, J. G. (1992), "Model Specification Tests and Artificial Regressions," Journal of Economic Literature, 30, 102-146.

Pindyck, R. S. and D. L. Rubinfeld (1991), Econometric Models \& Economic Forecasts, 3rd Edition, McGrawHill: New York. 
Table 1: Generalized MSE relative to true Rubin Data

\begin{tabular}{rrrrrrrr} 
Model/Sample Size & 20 & 114 & 456 & & 20 & 114 & 456 \\
\hline Square: & 1.32 & 1.20 & 0.99 & Exp: & 3.01 & $\mathbf{1 . 3 8}$ & $\mathbf{1 . 0 8}$ \\
& 1.84 & $\mathbf{1 . 2 1}$ & $\mathbf{0 . 9 9}$ & & 3.21 & 1.39 & 1.08 \\
& $\mathbf{2 . 0 4}$ & 1.34 & 0.99 & & $\mathbf{3 . 8 7}$ & 1.43 & 1.09 \\
& 3.33 & 1.47 & 1.49 & & 8.61 & 4.27 & 4.31 \\
Linear: & 1.04 & 1.08 & 0.99 & & & & \\
& 2.25 & 1.49 & $\mathbf{0 . 9 9}$ & & & & \\
& $\mathbf{2 . 5 7}$ & $\mathbf{1 . 5 5}$ & 0.99 & & & & \\
& 4.99 & 1.77 & 1.04 & & & &
\end{tabular}

key: uncorrected, $e^{2}, \tilde{\mathbf{e}}^{2}, \hat{\mathrm{e}}^{2}$ 
Table 2: Generalized MSE relative to true

Mix Data, with Intercept

\begin{tabular}{rrrrrrr} 
& \multicolumn{3}{c}{ All Variates } & \multicolumn{3}{c}{ Exact Variates } \\
Model/Sample Size & 20 & 114 & 456 & 20 & 114 & 456 \\
\hline Square: & 2.29 & 1.27 & 1.03 & $\mathbf{1 . 2 3}$ & 1.09 & 1.00 \\
& 2.32 & $\mathbf{1 . 2 8}$ & $\mathbf{1 . 0 3}$ & 1.25 & $\mathbf{1 . 0 9}$ & $\mathbf{1 . 0 0}$ \\
& $\mathbf{2 . 7 9}$ & 1.33 & 1.03 & 2.06 & 1.09 & 1.00 \\
& 7.75 & 2.42 & 2.36 & 2.29 & 2.42 & 2.36 \\
& & & & & \\
Exp: & 2.13 & $\mathbf{1 . 3 2}$ & $\mathbf{1 . 0 2}$ & $\mathbf{1 . 5 6}$ & $\mathbf{1 . 0 6}$ & $\mathbf{0 . 9 7}$ \\
& $\mathbf{2 . 6 4}$ & 1.32 & 1.02 & 1.58 & 1.06 & 0.97 \\
& $\mathbf{2 . 6 7}$ & 1.34 & 1.02 & 1.78 & 1.06 & 0.97 \\
& 4.54 & 2.03 & 2.03 & 2.13 & 2.03 & 2.06 \\
& & & & & & \\
Linear: & 1.16 & 1.19 & 1.08 & 1.07 & $\mathbf{0 . 9 8}$ & 1.04 \\
& 1.77 & 1.21 & $\mathbf{1 . 0 8}$ & $\mathbf{1 . 0 7}$ & 0.98 & $\mathbf{1 . 0 4}$ \\
& $\mathbf{2 . 0 5}$ & $\mathbf{1 . 2 3}$ & 1.08 & 1.11 & 0.98 & 1.04 \\
& 3.21 & 1.27 & 1.26 & 1.16 & 1.19 & 1.26
\end{tabular}

key: uncorrected, $e^{2}, \tilde{\mathbf{e}}^{2}, \hat{\mathrm{e}}^{2}$ 
Table 3: Generalized MSE relative to true

Trend Data, with Intercept

\begin{tabular}{cccccccc} 
& \multicolumn{3}{c}{ All Variates } & \multicolumn{3}{c}{ Exact Variates } \\
Model/Sample Size & 20 & 114 & 456 & & 20 & 114 & 456 \\
\hline Square: & 0.91 & 1.08 & 1.08 & 0.91 & 1.03 & 1.06 \\
& 1.23 & $\mathbf{1 . 0 9}$ & $\mathbf{1 . 0 8}$ & 1.08 & $\mathbf{1 . 0 3}$ & $\mathbf{1 . 0 6}$ \\
& $\mathbf{1 . 2 3}$ & 1.10 & 1.08 & $\mathbf{1 . 0 8}$ & 1.03 & 1.06 \\
& 1.61 & 1.13 & 2.18 & 1.22 & 1.13 & 2.18 \\
& & & & & & \\
Exp: & 1.00 & 1.01 & $\mathbf{1 . 1 0}$ & 1.00 & 1.01 & $\mathbf{1 . 0 8}$ \\
& 1.61 & $\mathbf{1 . 1 2}$ & 1.10 & 1.35 & 1.04 & 1.08 \\
& $\mathbf{1 . 6 2}$ & 1.12 & 1.10 & $\mathbf{1 . 3 5}$ & $\mathbf{1 . 0 4}$ & 1.08 \\
& 1.94 & 1.13 & 2.89 & 1.49 & 1.04 & 2.89 \\
& & & & & & \\
Linear: & 0.99 & 1.04 & 1.07 & & 0.99 & 1.04 & 1.05 \\
& 1.34 & 1.09 & $\mathbf{1 . 0 7}$ & 1.16 & 1.04 & $\mathbf{1 . 0 5}$ \\
& $\mathbf{1 . 3 8}$ & $\mathbf{1 . 1 0}$ & 1.08 & & $\mathbf{1 . 1 7}$ & $\mathbf{1 . 0 4}$ & 1.05 \\
& 1.58 & 1.11 & 1.25 & 1.26 & 1.04 & 1.25
\end{tabular}

key: uncorrected, $e^{2}, \tilde{\mathbf{e}}^{2}, \hat{\mathrm{e}}^{2}$ 
Table 4: Generalized MSE relative to true Mix Data, Proportionate

\begin{tabular}{rrrrrrr} 
& \multicolumn{3}{c}{ All Variates } & \multicolumn{3}{c}{ Exact Variates* } \\
Model/Sample Size & 20 & 114 & 456 & 20 & 114 & 456 \\
\hline Square: & $\mathbf{2 8 5}$ & $35 \mathrm{~K}$ & $1.2 \mathrm{e} 6$ & 0.94 & 1.01 & 1.00 \\
& 291 & $55 \mathrm{~K}$ & $\mathbf{1 . 4 e \mathbf { 6 }}$ & 2938 & $35 \mathrm{~K}$ & $31 \mathrm{~K}$ \\
& 467 & $\mathbf{7 2 K}$ & $1.5 e 6$ & & & \\
2938 & $279 \mathrm{~K}$ & $1.0 \mathrm{e} 7$ & & & \\
& & & & & & \\
Exp: & 2.13 & $\mathbf{1 . 3 2}$ & $\mathbf{1 . 0 2}$ & $\mathbf{1 . 7 7}$ & 1.66 & 1.62 \\
& 2.64 & 1.32 & 1.02 & 1.79 & $\mathbf{1 . 6 6}$ & $\mathbf{1 . 6 2}$ \\
& $\mathbf{2 . 6 7}$ & 1.34 & 1.02 & 2.09 & 1.72 & 1.63 \\
& 4.54 & 2.03 & 2.02 & 2.13 & 2.03 & 2.03 \\
& & & & & \\
Linear: & 2.97 & 1.35 & 1.20 & 0.92 & 0.97 & 1.02 \\
& $\mathbf{3 . 3 7}$ & $\mathbf{1 . 3 6}$ & $\mathbf{1 . 2 0}$ & 8.19 & 12.85 & 13.16 \\
& 4.37 & 1.38 & 1.20 & & & \\
& 6.24 & 5.43 & 5.78 & & & \\
* The three corrections give same results for Square and Linear cases
\end{tabular}


Table 6: Generalized MSE relative to true Rubin Data: Misspecified

Generated Square, Misspecified as

Exponential

\begin{tabular}{rrrrrr}
20 & 114 & 456 & 20 & 114 & 456 \\
\hline 1.32 & $\mathbf{1 . 3 6}$ & $\mathbf{1 . 0 3}$ & 1.32 & 1.47 & 1.28 \\
2.52 & 1.37 & 1.03 & 8.98 & 8.70 & $\mathbf{1 . 3 2}$ \\
$\mathbf{2 . 8 4}$ & 1.40 & 1.04 & $\mathbf{1 0 . 8 1}$ & $\mathbf{1 1 . 5 6}$ & 1.47 \\
6.28 & 1.47 & 1.49 & 47.66 & 21.35 & 1.49
\end{tabular}

Generated Exponential, Misspecified as

Square

Linear

\begin{tabular}{rrrrrr}
20 & 114 & 456 & 20 & 114 & 456 \\
\hline 3.01 & 4.17 & 1.33 & 3 & 4 & 4 \\
3.20 & 4.27 & $\mathbf{1 . 3 4}$ & 677 & 3706 & 4825 \\
$\mathbf{3 . 5 6}$ & $\mathbf{5 . 3 3}$ & 1.45 & $\mathbf{8 3 2}$ & 5168 & 5759 \\
6.54 & 17.16 & 4.31 & 3991 & $\mathbf{5 6 0 8}$ & $\mathbf{5 8 9 0}$
\end{tabular}

Generated Linear, Misspecified as

Square

Exponential

\begin{tabular}{rrrrrr}
20 & 114 & 456 & 20 & 114 & 456 \\
\hline 1.04 & 1.08 & 0.99 & 1.04 & 1.08 & 1.04 \\
1.60 & 1.20 & $\mathbf{0 . 9 9}$ & 2.29 & $\mathbf{1 . 3 9}$ & $\mathbf{1 . 0 5}$ \\
$\mathbf{1 . 7 2}$ & $\mathbf{1 . 2 1}$ & 0.99 & $\mathbf{2 . 4 8}$ & 1.39 & 1.05 \\
2.53 & 1.25 & 1.04 & 5.04 & 1.42 & 1.05
\end{tabular}

key: uncorrected, $e^{2}, \tilde{\mathbf{e}}^{2}, \hat{\mathrm{e}}^{2}$ 
Table 7: Generalized MSE relative to true

Mix Data, with Intercept: Misspecified

Generated Square, Misspecified as

Exponential Linear

\begin{tabular}{rrrrrr}
20 & 114 & 456 & 20 & 114 & 456 \\
\hline 2.29 & $\mathbf{1 . 3 7}$ & $\mathbf{1 . 0 7}$ & 2.29 & 2.42 & 1.37 \\
2.57 & 1.37 & 1.07 & 3.45 & 2.86 & $\mathbf{1 . 3 8}$ \\
$\mathbf{2 . 5 9}$ & 1.39 & 1.07 & $\mathbf{5 . 0 6}$ & $\mathbf{3 . 0 7}$ & 1.42 \\
4.40 & 2.42 & 2.35 & 10.22 & 3.67 & 2.36
\end{tabular}

Generated Exponential, Misspecified as

\begin{tabular}{rccrcc}
\multicolumn{3}{c}{ Square } & \multicolumn{3}{c}{ Linear } \\
20 & 114 & 456 & 20 & 114 & 456 \\
\hline 2.13 & 1.21 & 1.01 & 2.13 & 2.03 & 1.49 \\
2.28 & $\mathbf{1 . 2 2}$ & $\mathbf{1 . 0 1}$ & 6.87 & 5.66 & $\mathbf{1 . 5 2}$ \\
$\mathbf{2 . 6 3}$ & 1.32 & 1.01 & $\mathbf{1 4 . 5 3}$ & $\mathbf{6 . 4 7}$ & 1.62 \\
$\mathbf{6 . 7 4}$ & 2.03 & 2.02 & 45.42 & 9.98 & 2.03
\end{tabular}

Generated Linear, Misspecified as

Square Exponential

\begin{tabular}{rrrrrr}
20 & 114 & 456 & 20 & 114 & 456 \\
\hline 1.16 & 1.10 & 1.07 & 1.16 & 1.19 & 1.11 \\
1.60 & $\mathbf{1 . 1 0}$ & $\mathbf{1 . 0 7}$ & 2.19 & $\mathbf{1 . 2 5}$ & $\mathbf{1 . 1 1}$ \\
$\mathbf{1 . 8 6}$ & 1.11 & 1.07 & $\mathbf{2 . 2 1}$ & 1.25 & 1.11 \\
3.16 & 1.19 & 1.26 & 3.58 & 1.27 & 1.27
\end{tabular}

key: uncorrected, $e^{2}, \tilde{\mathbf{e}}^{2}, \hat{\mathrm{e}}^{2}$ 
Table 8: Generalized MSE relative to true

Mix Data, Proportionate: Misspecified

Generated Square, Misspecified as

Exponential Linear*

\begin{tabular}{rrrrrr}
20 & 114 & 456 & 20 & 114 & 456 \\
\hline $3 \mathrm{e} 3$ & $35 \mathrm{e} 3$ & $31 \mathrm{e} 3$ & 5.43 & 30.88 & 28.15 \\
$\mathbf{9 e 3}$ & $\mathbf{1 6 e} 4$ & $\mathbf{1 7 e} 4$ & $3 \mathrm{e} 3$ & $3 \mathrm{e} 4$ & $3 \mathrm{e} 4$ \\
$10 \mathrm{e} 3$ & $16 \mathrm{e} 4$ & $17 \mathrm{e} 4$ & & & \\
$15 \mathrm{e} 3$ & $17 \mathrm{e} 4$ & 1 \%e4 & & &
\end{tabular}

Generated Exponential, Misspecified as

\begin{tabular}{rrrrrr}
\multicolumn{3}{c}{ Square* $^{*}$} & \multicolumn{3}{c}{ Linear* } \\
20 & 114 & 456 & 20 & 114 & 456 \\
\hline 2.13 & 2.03 & 2.02 & 1.47 & 2.03 & 2.03 \\
67.49 & $7 e 3$ & $9 e 3$ & 2.12 & 4.04 & 4.02
\end{tabular}

Generated Linear, Misspecified as Square* Exponential

\begin{tabular}{rrrrrr}
20 & 114 & 456 & 20 & 114 & 456 \\
\hline 7.51 & 12.86 & 13.16 & 8.19 & 12.85 & 13.16 \\
8.19 & 38.13 & 43.55 & $\mathbf{3 3 . 4 4}$ & $\mathbf{5 9 . 9 2}$ & $\mathbf{6 3 . 0 0}$ \\
& & & 40.29 & 61.06 & 63.29 \\
& & & 50.57 & 64.90 & 64.34
\end{tabular}

key: uncorrected, $e^{2}, \tilde{\mathbf{e}}^{2}, \hat{\mathrm{e}}^{2}$

* The three corrections give same results for Square and Linear cases. 
Table 9: Generalized MSE relative to true

Trend Data, Proportionate: Misspecified

Generated Square, Misspecified as

Exponential Linear*

\begin{tabular}{rrrrrr}
20 & 114 & 456 & 20 & 114 & 456 \\
\hline 59 & 269 & 1213 & 2.82 & 6.10 & 13.80 \\
$\mathbf{2 0 e 4}$ & $\mathbf{4 9 e 4}$ & $\mathbf{4 3 6 8}$ & 59 & 269 & 1214 \\
$26 e 4$ & $53 e 4$ & 4383 & & & \\
$21 \mathrm{e} 5$ & $76 \mathrm{e} 4$ & 4452 & & &
\end{tabular}

Generated Exponential, Misspecified as

\begin{tabular}{rrrrrr}
\multicolumn{3}{c}{ Square* $^{*}$} & \multicolumn{3}{c}{ Linear * $^{*}$} \\
20 & 114 & 456 & 20 & 114 & 456 \\
\hline 1.00 & 1.01 & 2.89 & 1.00 & 1.01 & 2.89 \\
51 & 2105 & 8074 & 2.83 & 3.86 & 4.68
\end{tabular}

Generated Linear, Misspecified as Square* Exponential

\begin{tabular}{rrrrrr}
20 & 114 & 456 & 20 & 114 & 456 \\
\hline 2.61 & 4.48 & 6.40 & 3.14 & 4.48 & 6.40 \\
3.14 & 8.23 & 19.29 & $\mathbf{2 2 2}$ & $\mathbf{2 2 2}$ & $\mathbf{2 5 . 9 5}$ \\
& & & 270 & 238 & 26.09 \\
& & & 585 & 260 & $\mathbf{2 6 . 3 4}$
\end{tabular}

key: uncorrected, $e^{2}, \tilde{\mathbf{e}}^{2}, \hat{\mathrm{e}}^{2}$

* The three corrections give same results for Square and Linear cases. 
Table 5: Generalized MSE relative to true Trend Data: Proportionate Cases

\begin{tabular}{|c|c|c|c|c|c|c|c|c|c|c|}
\hline \multirow[b]{2}{*}{ Model/Sample Size } & \multicolumn{3}{|c|}{ All Variates } & \multicolumn{3}{|c|}{ Partial Variates } & \multicolumn{4}{|c|}{ Exact Variates $^{a}$} \\
\hline & 20 & 114 & 456 & 20 & 114 & 456 & 20 & 114 & 456 & $456^{b}$ \\
\hline Square: & 10.01 & 45 & 1215 & 5.55 & 42 & 1215 & 0.9 & 0.95 & 1.08 & 1.02 \\
\hline & 10.50 & 62 & 1603 & 5.91 & 49 & 1636 & 59.01 & 269 & 1215 & 264 \\
\hline & 21.97 & 190 & 2090 & 6.28 & 168 & 1829 & & & & \\
\hline & 59.01 & 269 & 5240 & 59.01 & 269 & 6935 & & & & \\
\hline Exp: & 1.00 & 1.01 & 1.10 & 1.00 & 1.01 & 1.08 & 1.00 & 1.01 & 1.96 & 1.06 \\
\hline & 1.61 & 1.12 & 1.10 & 1.35 & 1.04 & 1.08 & 1.56 & 1.86 & 1.96 & 1.91 \\
\hline & 1.62 & 1.12 & 1.10 & 1.36 & 1.04 & 1.08 & 1.71 & 1.88 & 1.98 & 1.91 \\
\hline & 1.94 & 1.13 & 2.89 & 1.49 & 1.04 & 2.89 & 1.77 & 1.92 & 2.89 & 1.93 \\
\hline Linear: & 1.01 & 1.10 & 1.15 & 1.01 & 1.06 & 1.11 & 0.95 & 1.00 & 1.05 & 1.03 \\
\hline & 1.07 & 1.11 & 1.15 & 1.04 & 1.06 & 1.11 & 3.14 & 4.48 & 6.40 & 4.68 \\
\hline & 1.09 & 1.11 & 1.16 & 1.05 & 1.06 & 1.11 & & & & \\
\hline & 1.10 & 1.70 & 3.22 & 1.06 & 1.70 & 3.22 & & & & \\
\hline
\end{tabular}

